\title{
Identification of a four-long non-coding RNA signature in predicting breast cancer survival
}

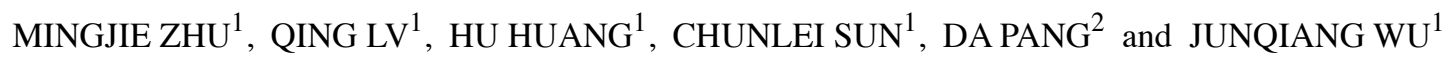 \\ ${ }^{1}$ Department of Breast Surgery, The Affiliated Hospital of Jiangnan University, Wuxi, Jiangsu 214035; \\ ${ }^{2}$ Department of Breast Surgery, Harbin Medical University Cancer Hospital, Harbin, Heilongjiang 150081, P.R. China
}

Received January 3, 2019; Accepted October 4, 2019

DOI: $10.3892 / 01.2019 .11063$

\begin{abstract}
Long non-coding RNAs (lncRNAs) serve key roles in tumorigenesis and are differentially expressed in cancer. Using bioinformatics and statistical methods, the present study aimed to identify an lncRNA signature to predict breast cancer survival. The gene expression data of 768 patients with breast cancer were downloaded from The Cancer Genome Atlas database, and Cox regression, Kaplan-Meier and receiver operating characteristic (ROC) analyses were performed to construct and validate a predictive model. Gene Ontology term enrichment and Kyoto Encyclopedia of Genes and Genomes pathway analysis were employed to predict the functions of the indicated lncRNAs. A signature consisting of four lncRNAs, including PVT1, MAPT-AS1, LINC00667 and LINC00938, was identified, and patients were subsequently divided into high- and low-risk groups according to the median risk score. Kaplan-Meier analysis confirmed that patients in the high-risk group exhibited significantly poorer overall survival rate in both the training $(\mathrm{P}=0.0151)$ and the validation set $(\mathrm{P}=0.0016)$; furthermore, ROC analysis confirmed that the model could predict patient survival with a certain sensitivity and specificity. In conclusion, the four-lncRNA signature presents a potential prognostic biomarker for breast cancer that may be relevant for clinical application.
\end{abstract}

Correspondence to: Dr Junqiang Wu, Department of Breast Surgery, The Affiliated Hospital of Jiangnan University, 200 Huihe Road, Wuxi, Jiangsu 214035, P.R. China

E-mail: dr_junqiang_wu@163.com

Abbreviations: KEGG, Kyoto Encyclopedia of Genes and Genomes; lncRNA, long non-coding RNA; NSCLC, non-small cell lung cancer; OS, overall survival; ROC, receiver operating characteristic; SIS, sure independence screening; TCGA, The Cancer Genome Atlas

Key words: breast cancer, long non-coding RNA, overall survival, prognostic predictor

\section{Introduction}

Breast cancer is one of the most common malignancies in women in both developed and developing countries, and each year $>1,300,000$ cases of breast cancer are reported globally (1). For decades, clinicopathological features have been utilized to evaluate the prognosis of patients with breast cancer, including tumor size, clinical stage, intrinsic subtype and lymph node status (2-5). However, these factors are limited in their prognostic capability and are only useful in a number of patients (6). It is widely accepted that the underlying molecular mechanisms of breast cancer are complex and may involve the alterations of specific genomic regions, as well as epigenetic modifications in mammary epithelial cells $(7,8)$. To the best of our knowledge, patients with similar disease characteristics, who have received similar treatments may present with markedly different clinical outcomes. Therefore, accurately predicting patient outcome and subsequently selecting the appropriate treatment, reducing morbidity and prolonging survival time are of great clinical importance.

Long non-coding RNAs (lncRNAs) are a class of RNAs with $>200$ nucleotides and no known protein coding capability $(9,10)$. IncRNAs have been implicated in a wide range of biological processes, including tumor-suppressor modulation, RNA-RNA interactions, and epigenetic and post-transcriptional regulation (11-14). As additional biological functions of lncRNAs are identified, they have become the focus of an increasing number of studies. These studies have revealed that lncRNAs serve a role in carcinogenesis and possess specific expression patterns in cancer (15-17). To date, several lncRNAs have been regarded as diagnostic and/or prognostic biomarkers for specific malignancies, such as lncRNA HOX transcript antisense RNA (HOTAIR), which is overexpressed in breast cancer, thus promoting cancer cell invasion and metastasis by altering the methylation and gene expression of histone $\mathrm{H} 3 \mathrm{~K} 27$ via polycomb repressive complex 2 (18). Another lncRNA, metastasis-associated lung adenocarcinoma transcript 1 , was first identified in non-small cell lung cancer (NSCLC); its high level of expression was strongly correlated with an increased risk of NSCLC, and was associated with metastasis and poor patient outcome (19).

However, the predictive ability of single lncRNAs is still unsatisfactory, resulting in high numbers of both false positive and negative results (20). Therefore, the present study 
aimed to identify a four-lncRNA signature able to predict the overall survival (OS) rate of patients with breast cancer, and to validate the prognostic value of the identified lncRNAs using high-throughput sequencing data from The Cancer Genome Atlas (TCGA) database.

\section{Materials and methods}

Breast cancer gene expression data from TCGA and Gene Expression Omnibus (GEO) databases. Breast cancer gene expression data, including coding and non-coding RNA sequence data, were acquired from TCGA (https://cancergenome.nih.gov/) together with the corresponding clinical information. Until 2017, 1,098 breast cancer samples were available from TCGA, though in the present study only those including patient survival status were selected $(n=768)$; this enabled the determination of any association between the expression of lncRNAs of the lncRNA-expression signature and the corresponding OS time for breast cancer. These 768 breast cancer samples were divided equally into a training set (to identify the gene expression signature) and a validation set (to validate the gene expression signature). To confirm the expression levels of the differentially expressed genes, the gene expression dataset GSE5764 (21), containing 10 breast cancer tissue samples and 20 non-cancerous samples, was downloaded from GEO (https://www.ncbi.nlm.nih.gov/geo/; Affymetrix GPL570 platform, Affymetrix Human Genome U133 Plus 2.0 Array; Affymetrix; Thermo Fisher Scientific, Inc.).

Identification of lncRNAs. RNA genes downloaded from TCGA were compared with published lncRNAs from the MiTranscriptome database (http://mitranscriptome.org/). Potential lncRNAs were identified as transcriptome sequences that were mapped to corresponding lncRNAs, rather than any protein-coding region, and were not identified as protein-coding genes in the National Center for Biotechnology Information database (https://www.ncbi.nlm.nih.gov/).

Gene expression data analysis. Raw read counts of the transcriptomic data from TCGA were normalized using the quartile normalization method and logarithmically transformed to a normal distribution. The Bioconductor package DESeq2 (https://www.bioconductor.org/packages/release/bioc/html/DESeq2.html, version 1.24.0) was used to perform the normalization and identify differentially expressed genes in breast cancer samples compared with adjacent normal tissues, with an adjusted $\mathrm{P}<0.05$ and an absolute $\log 2$-based fold-change $>0.5$. For gene expression data from GEO, the R package limma (https://www.bioconductor. org/packages/release/bioc/html/limma.html, version 3.40.6) was used to conduct differential expression analysis.

Establishment of a prognostic signature. To establish a prognostic signature for breast cancer, a two-step method was employed using the R package SIS (https://CRAN.R-project. org/package $=$ SIS, version 0.8-6) for sure independence screening. Firstly, univariate Cox regression analysis was performed to identify survival-associated genes. Secondly, SIS (based on the least absolute shrinkage and selection operator, Cox-penalized regression model) was used to identify important variables and construct multi-gene-based prognostic signatures for OS rate prediction.

Guilt by association analysis. To identify genes that correlated with the four lncRNAs of the prognostic signature, data from TCGA were used to evaluate the pairwise Pearson's correlation between the expression levels of the target lncRNAs. Only associated genes with an absolute $r \geq 0.3$ and a significant correlation $(\mathrm{P}<0.05)$ were retained. Gene Ontology $(\mathrm{GO})$ and Kyoto Encyclopedia of Genes and Genomes (KEGG; https://www. genome.jp/kegg/) pathway analyses were performed using the Database for Annotation, Visualization and Integrated Discovery (DAVID; https://david.ncifcrf.gov/).

Statistical analysis. Kaplan-Meier analysis was used to estimate the performance of the prognostic signatures, and log-rank test was performed to evaluate statistical significance. A risk score was calculated for each patient according to the formula of the four-lncRNA signature, and patients were divided into high- and low-risk groups using the median score as a cut-off. Receiver operating characteristic (ROC) analysis was used to evaluate the sensitivity and specificity of the four-lncRNA signature and other biomarkers, including TP53, MKI67, ESR1, PGR, ERBB2 and HOTAIR. All statistical analyses were conducted using R 3.5.2 (https://www.r-project. org/), and $\mathrm{P}<0.05$ was considered to indicate a statistically significant difference.

\section{Results}

Patient characteristics. All 768 patients were diagnosed with breast cancer based on clinicopathological evaluation. The clinical stage and histological subtype were determined using the Tumor-Node-Metastasis staging (22) and immunohistochemical molecular typing methods, respectively. According to the data, the estrogen receptor (ER), progesterone receptor (PR) and human epidermal growth factor receptor 2 (HER2) status of each patient was indicated as positive, negative or indeterminate. In addition, the ranges of the OS and relapse-free survival times were 1-8,605 and 1-8,556 days, respectively. Patient characteristics are displayed in Table I.

Differential expression analysis and determination of the four-lncRNA signature in the training set. Differential expression analysis was employed to select differentially expressed RNAs in normal and cancerous tissues; a total of 8,854 upregulated and 5,939 downregulated RNAs were identified. Subsequently, all possible combinations of four lncRNAs were analyzed and compared using the two-step Cox regression method. A total of 7 models consisting of four lncRNAs were identified (Table SI); among these candidates, one model was identified as the most suitable for predicting the OS of patients with breast cancer. Patients were divided into high- and low-risk groups using the median risk score as a cut-off, with the risk score calculated as follows: Risk score $=(-0.015 \mathrm{x}$ expression value of $P V T 1)+(-0.193 \mathrm{x}$ expression value of MAPT-AS1) + $(-0.116 \mathrm{x}$ expression value of $L I N C 00667)+(0.098 \mathrm{x}$ expression value of $L I N C 00938)$. The coefficients of this formula were derived from multivariate Cox regression analysis (Table SI). Kaplan-Meier analysis was also performed to determine the 
Table I. Demographic characteristics of the 768 patients with breast cancer included in the present study.

\begin{tabular}{|c|c|c|c|}
\hline Characteristics & Training set $(n=384)$ & Validation set $(n=384)$ & Total set $(n=768), \%$ \\
\hline \multicolumn{4}{|l|}{ Sex } \\
\hline Male & 4 & 3 & $7(0.91)$ \\
\hline Female & 380 & 381 & $761(99.09)$ \\
\hline \multicolumn{4}{|l|}{ TNM stage (22) } \\
\hline Stage I & 66 & 67 & $133(17.32)$ \\
\hline Stage II & 226 & 223 & $449(58.46)$ \\
\hline Stage III & 85 & 86 & $171(22.27)$ \\
\hline Stage IV & 7 & 8 & $15(1.95)$ \\
\hline \multicolumn{4}{|l|}{ ER status } \\
\hline Negative & 90 & 92 & $182(23.70)$ \\
\hline Positive & 291 & 290 & $581(75.65)$ \\
\hline Indeterminate & 3 & 2 & $5(0.65)$ \\
\hline \multicolumn{4}{|l|}{ PR status } \\
\hline Negative & 125 & 128 & $253(32.94)$ \\
\hline Positive & 256 & 254 & $510(66.41)$ \\
\hline Indeterminate & 3 & 2 & $5(0.65)$ \\
\hline \multicolumn{4}{|l|}{ HER2 status } \\
\hline Negative & 228 & 231 & $459(59.77)$ \\
\hline Positive & 92 & 87 & $179(23.31)$ \\
\hline Indeterminate & 64 & 66 & $130(16.93)$ \\
\hline \multicolumn{4}{|l|}{ Vital status } \\
\hline Alive & 326 & 326 & $652(84.90)$ \\
\hline Deceased & 58 & 58 & $116(15.10)$ \\
\hline OS time (range), days & $7-8,556$ & $1-8,605$ & $1-8,605$ \\
\hline \multicolumn{4}{|l|}{ RFS status } \\
\hline Relapsed & 295 & 288 & $583(75.91)$ \\
\hline Relapse-free & 89 & 96 & $185(24.09)$ \\
\hline RFS time (range), days & $7-8,556$ & $1-8,391$ & $1-8,556$ \\
\hline
\end{tabular}

TNM, Tumor-Node-Metastasis; RFS, relapse-free survival; OS, overall survival; HER2, human epidermal growth factor receptor 2; $\mathrm{ER}$, estrogen receptor; PR, progesterone receptor.

association between the expression levels of the four-lncRNA signature and patient OS. Compared with those of the low-risk group, high-risk patients exhibited significantly poorer OS rate (log-rank $\mathrm{P}=0.0151$; Fig. 1A).

Validation of the four-lncRNA signature in the validation set. To confirm the predictive capacity of the four-lncRNA signature identified in the training set, the equivalent analyses were also performed in the validation set. Patients were divided into low- and high-risk groups, and the differences between patient OS rates were compared using Kaplan-Meier analysis. Patients in the high-risk group possessed significantly lower OS rate than those of patients in the low-risk group (log-rank $\mathrm{P}=0.0016$; Fig. 1B), which was consistent with the findings from the training set. Furthermore, ROC analysis was performed to evaluate the sensitivity and specificity of survival prediction; the area under the curve (AUC) was 0.641 (Fig. 2A), indicating that the four-lncRNA signature was able to accurately predict the survival of patients with breast cancer.
Four-lncRNA signature in different clinical stages and molecular subtypes. ROC analyses were performed to investigate whether the four-lncRNA signature was applicable to different breast cancer stages and molecular subtypes. In stages I-IV, the AUC values were $0.595,0.687,0.634$ and 0.645 , respectively (Fig. S1), indicating that the four-lncRNA signature was able to predict the survival of patients at different clinical stages of breast cancer. Regarding subtype, the AUC values in the four molecular subgroups were 0.637, 0.654, 0.688 and 0.613, respectively (Fig. S2), suggesting that the four-lncRNA signature served as a prognostic indicator for patients with different breast cancer subtypes.

Performance of the four-IncRNA signature compared with that of known biomarkers and individual lncRNAs. For further clarification, the performance of the four-lncRNA signature was compared with that of several known breast cancer biomarkers, including TP53, MKI67, ESR1, PGR, ERBB2 and HOTAIR, using ROC and Kaplan-Meier analyses. The sensitivity and 
A

Training set

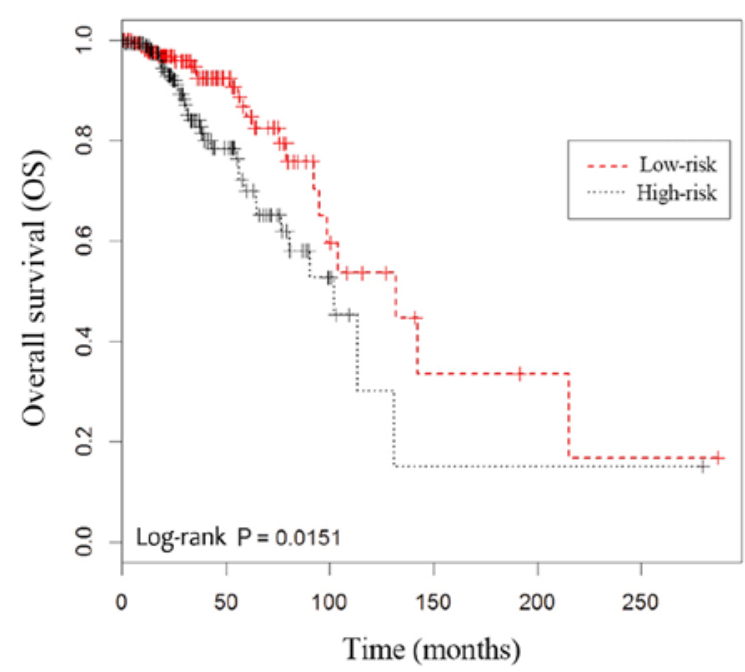

B

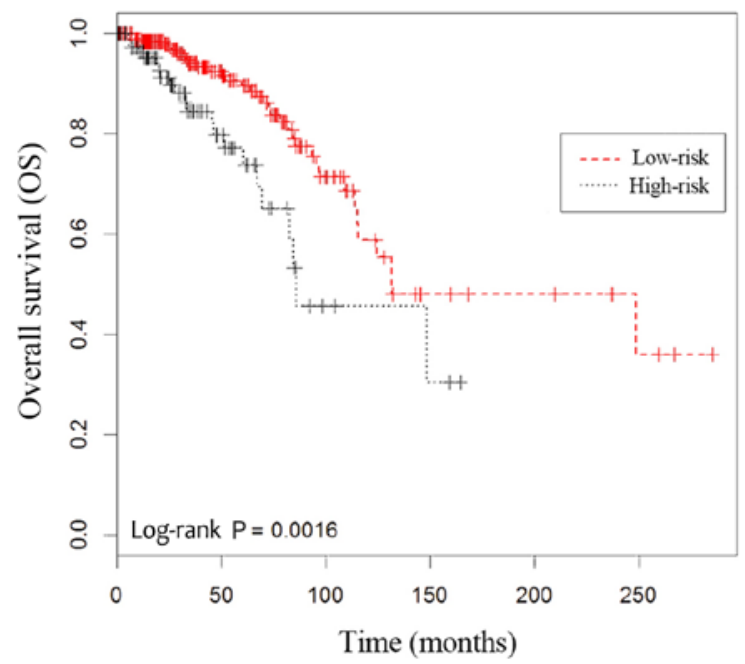

Figure 1. Kaplan-Meier analysis of overall survival of patients using the four-long non-coding RNA signature. Kaplan-Meier curves for (A) the training-set patients $(n=384)$ and $(B)$ the validation-set patients $(n=384)$. Two-sided log-rank test was performed to evaluate the survival differences between the two curves.
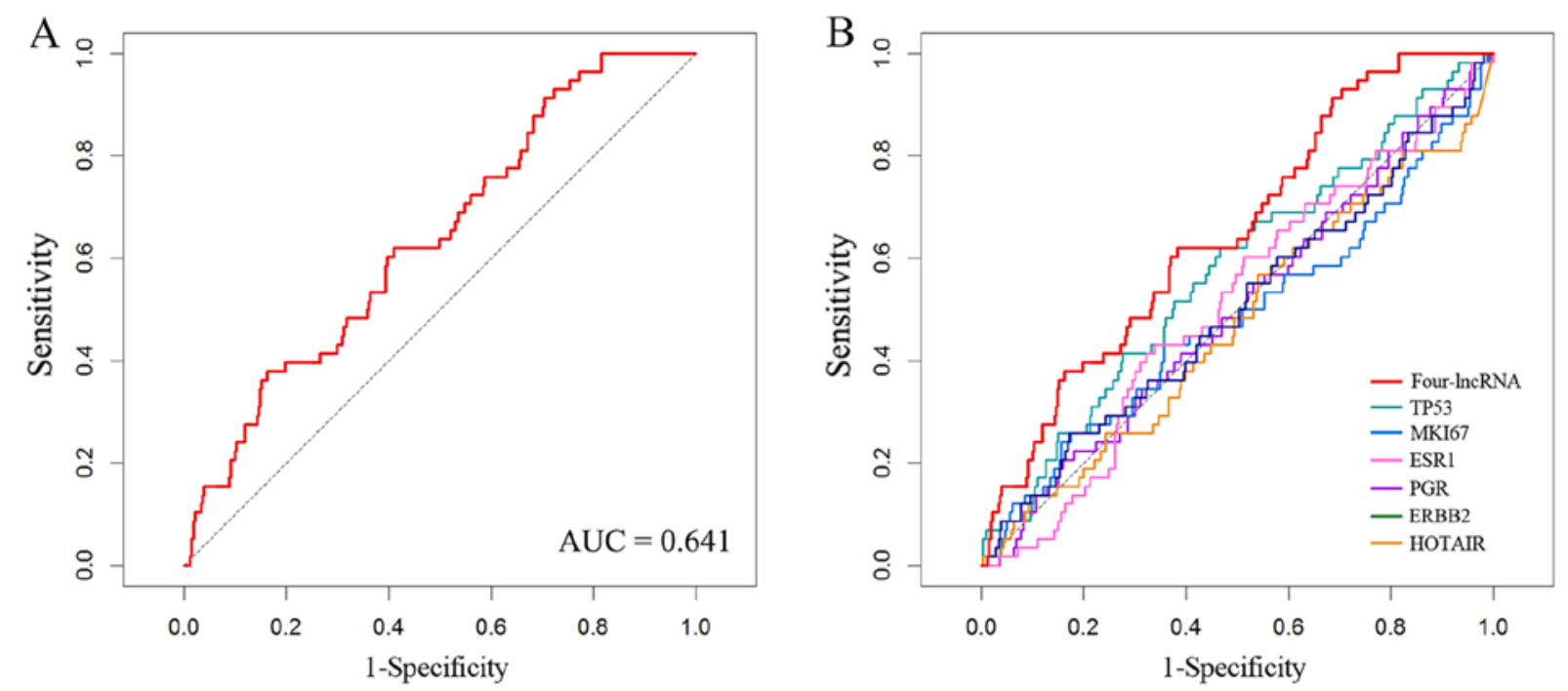

Figure 2. ROC analysis shows the sensitivity and specificity of the biomarkers in predicting the overall survival of patients. (A) ROC curves of the four-lncRNA signature. (B) ROC curves of several known biomarkers: TP53, MKI67, ESR1, PGR, ERBB2 and HOTAIR. IncRNA, long non-coding RNA; ROC, receiver operating characteristic.

specificity of these six known biomarkers are displayed in Fig. 2B. The AUC values of TP53, MKI67, ESR1, PGR, ERBB2 and HOTAIR were $0.574,0.483,0.510,0.501,0.501$ and 0.473 (data not shown), respectively, while the AUC value (0.641) of the four-lncRNA signature was greater. Kaplan-Meier analysis revealed that only TP53 was significantly associated with patient OS (Fig. 3A; log-rank $\mathrm{P}=0.0396$ ), while the other selected biomarkers were not (Fig. 3B-F). Moreover, the four IncRNAs from the identified model were also evaluated. ROC curve analysis generated AUC values for PVT1, MAPT-AS1, LINC00667 and LINC00938 as 0.532, 0.553, 0.550 and 0.480, respectively (Fig. S3), which were smaller than that of the four-lncRNA signature. In addition, the results of Kaplan-Meier analysis indicated that the differential expression of these lncRNAs was not significantly associated with the OS rate of patients with breast cancer (log-rank P>0.05; Fig. S4).
Relative expression levels and potential biological functions of the four lncRNAs of the lncRNA signature. To further investigate the potential functions of the four lncRNAs of the signature, gene expression data from the GSE5764 dataset were downloaded from the GEO database, and differential expression analysis was performed. The fold-change values of PVT1, MAPT-AS1, LINC00667 and LINC00938 were 2.031, 3.057, 1.579, 0.455, respectively. This result indicated that PVT1, MAPT-AS1 and LINC00667 were upregulated in breast cancer tissues, and that LINC00938 was downregulated. GO enrichment and KEGG pathway analyses were conducted. According to the results of GO analysis, the primary $P V T 1$-associated functions were 'transcription elongation', 'mitochondrial electron transport' and 'endoplasmic reticulum-associated degradation' (Fig. 4A). MAPT-AS1 was associated with 'cilium morphogenesis' and 'cilium 
A

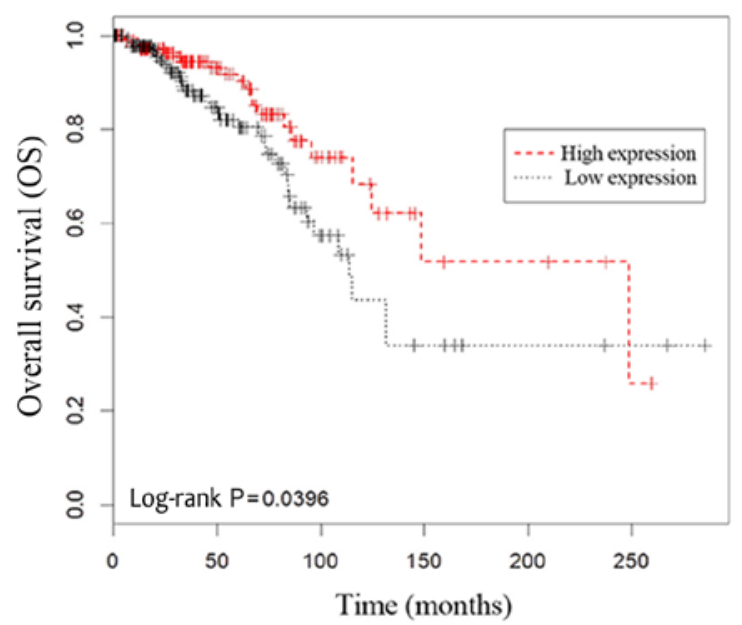

C

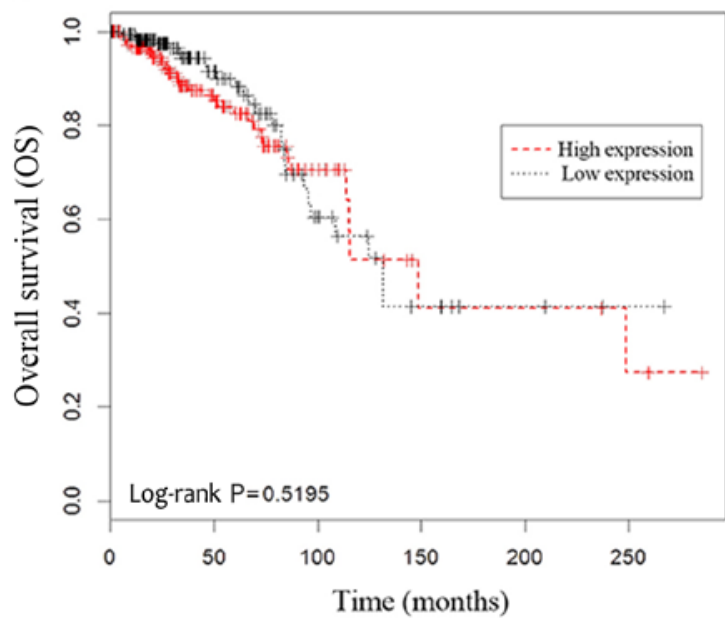

E

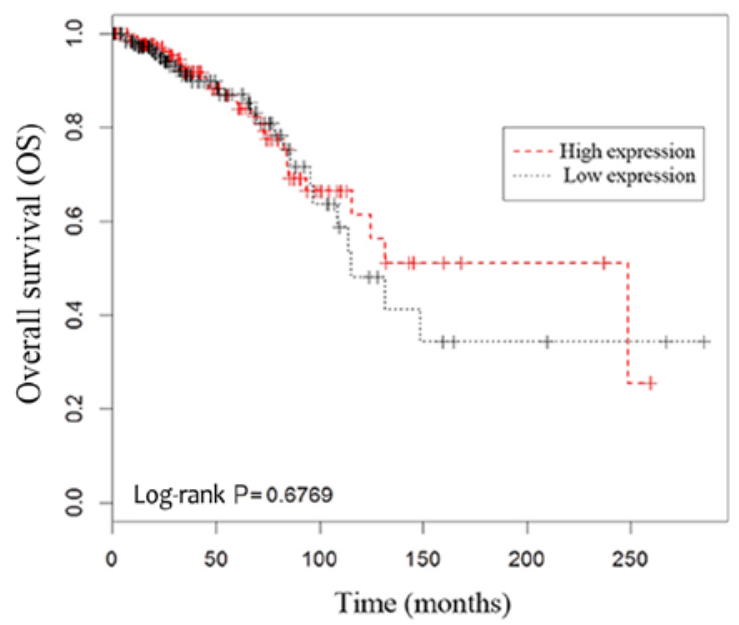

B

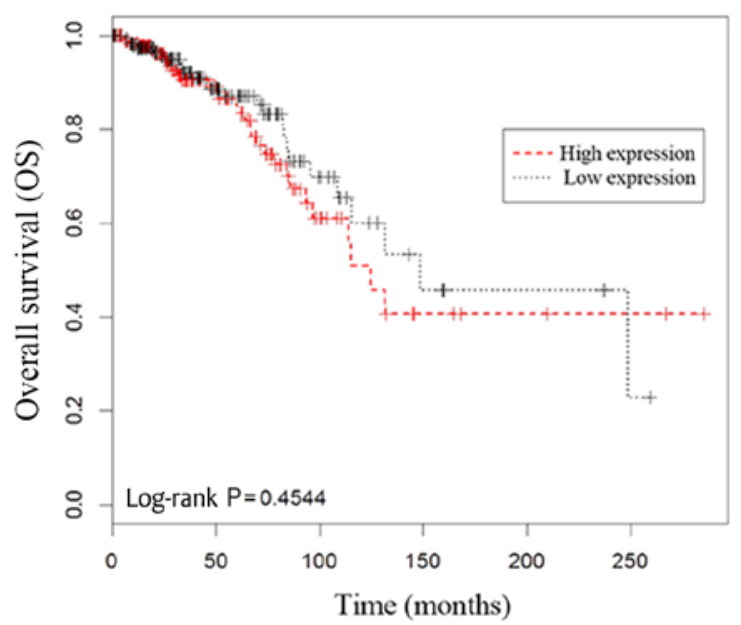

D

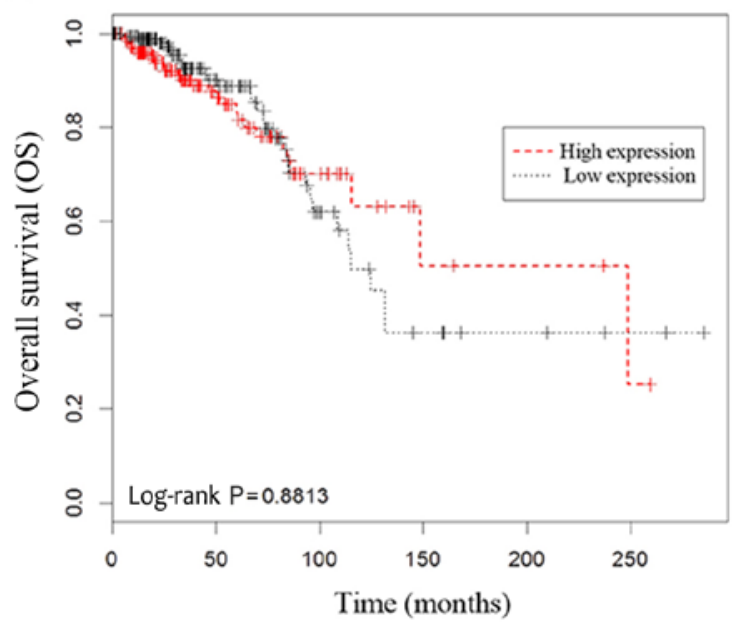

F

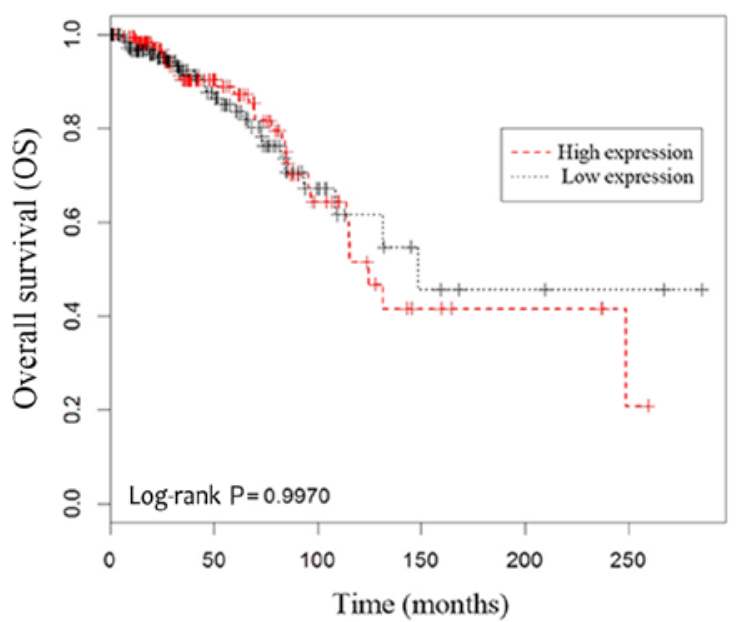

Figure 3. Kaplan-Meier analysis of different known biomarkers for patients in the validation set. (A) TP53 expression and overall survival of patients. (B) MKI67 expression and overall survival of patients. (C) ESR1 expression and overall survival rate of patients. (D) $P G R$ expression and overall survival rate of patients. (E) ERBB2 expression and overall survival rate of patients. (F) HOTAIR expression and overall survival rate of patients. HOTAIR, HOX transcript antisense RNA; PGR, progesterone receptor.

assembly', 'intraciliary retrograde transport' and 'neurological system process' (Fig. 4B). LINC00667 was associated with 'regulation of transcription, DNA-templated', 'centrosome organization' and 'regulation of cell morphogenesis' (Fig. 4C), and LINC00983 was associated with biological processes involved in 'cilium assembly' and 'S-adenosylmethionine metabolic process' (Fig. 4D). Moreover, KEGG analysis also indicated several biological processes and pathways that 


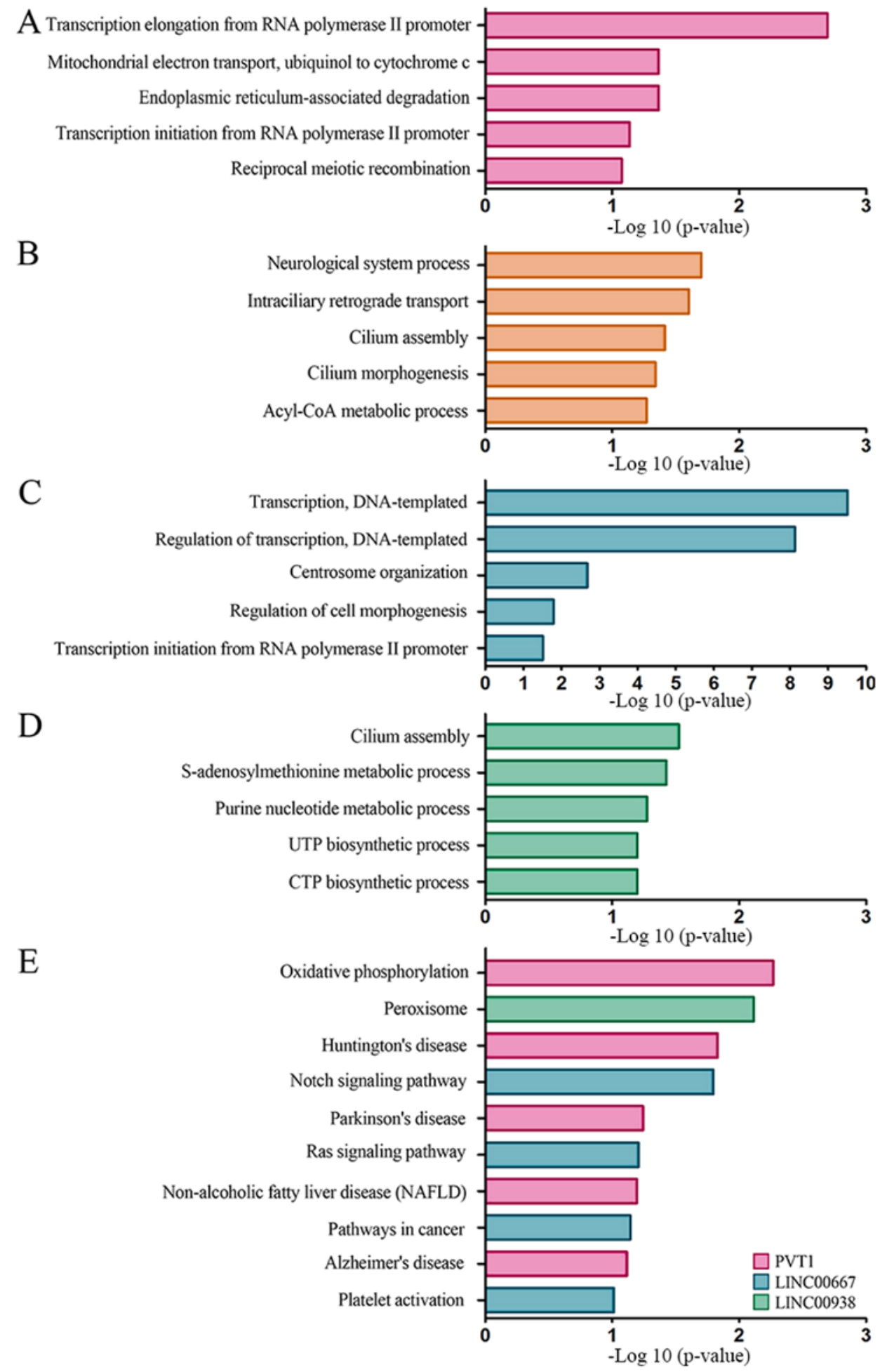

Figure 4. Potential biological functions of the four lncRNAs from the four-lncRNA signature. (A) GO analysis for PVT1. (B) GO analysis for MAPT-AS1. (C) GO analysis for LINC00667. (D) GO analysis for LINC00938. (E) Kyoto Encyclopedia of Genes and Genomes analysis for the four lncRNAs. GO, Gene Ontology; lncRNA, long non-coding RNA.

potentially associated with the signature lncRNAs, including 'Notch signaling pathway', 'oxidative phosphorylation' and 'Huntington's disease' (Fig. 4E).

\section{Discussion}

In the present study, the RNA expression data of 768 patients from TCGA were analyzed, and 14,793 RNAs that were differently expressed in normal vs. cancerous tissues were selected. Following multivariate Cox regression analysis (data not shown), a model consisting of four lncRNAs ( $P V T 1$, MAPT-AS1, LINC00667 and LINC00938) was determined to predict the survival of patients with breast cancer, while Kaplan-Meier and ROC analyses confirmed that this model was able to predict OS to an acceptable degree of specificity and sensitivity. 
In previous years, an increasing number of lncRNAs have been identified and are widely considered to be a novel class of gene regulators in various types of cancer $(11,23)$. With the development of high-throughput sequencing, a gradually increasing number of sequencing data have been used to study cancer-associated lncRNAs. Using transcriptome sequencing, Zou et al (24) identified two novel lncRNAs, LCE5A-1 and KCTD6-3, that are associated with head and neck carcinogenesis, and Ylipää et al (25) established prostate cancer associated transcript-1 as a novel oncogenic lncRNA, confirming its association with castration-resistant prostate cancers. To date, aberrant lncRNA expression levels have been observed in numerous cancer types, making these lncRNAs novel and reliable biomarkers for cancer diagnosis and prognosis $(26,27)$. The present study profiled the expression of lncRNAs associated with breast cancer prognosis using next-generation sequencing data, and determined a set of four lncRNAs that, when combined, may be used as a potential biomarker for the prognosis of breast cancer.

Previous studies have identified various biomarkers with prognostic value in breast cancer. TP53 is a recognized tumor-suppressor gene, the encoded protein of which responds to a diverse range of cellular stimuli to regulate the expression of target genes. Mutations in these genes are associated with a variety of human cancers, such as gastric cancer, colorectal cancer and breast cancer (28). MKI67 is a nucleoprotein-coding gene; its expression product, $\mathrm{Ki}-67$, has been identified as a biomarker of cell proliferation, which is regarded as a predictor of patient outcome (29). Additionally, IncRNA HOTAIR has been confirmed to promote breast cancer invasion and metastasis (30). The roles of ESR I, PGR and $E R B B 2$, also known as $E R, P R$ and $H E R 2$, respectively, have been widely recognized for breast cancer molecular typing and prognostics (31). In the present study, the prognostic value of several commonly used clinical prognostic molecular indicators was evaluated using ROC analysis and was compared with that of the four-lncRNA signature. ESRI, $P G R, E R B B 2, M K I 67$ and TP53 were all included in the analysis, and the result showed that the AUC value of the four-lncRNA signature was greater than that of the aforementioned biomarkers, which confirmed the four lncRNA signature as a potentially superior prognostic predictor. Additionally, according to the results of Kaplan-Meier analysis, the four individual lncRNAs of the signature were not adequate as independent prognostic predictors. This showed that, although a particular lncRNA may be associated with breast cancer, it may not reliably predict patient survival. However, the combination of these four lncRNAs was able to predict the outcomes of patients with breast cancer with satisfactory sensitivity and specificity.

lncRNAs are expressed at numerous cellular locations and fulfill a wide variety of regulatory roles at almost all stages of gene expression (32). Although specific lncRNAs have been implicated in a number of biological processes, the majority of their functions are not fully understood. Of the four lncRNAs of the signature, PVT1 is located on chromosome 8q24.21; a previous study has demonstrated that supernumerary copies of this chromosomal region are associated with various types of cancer, including breast and ovarian cancer, acute myeloid leukemia and Hodgkin's lymphoma (33). MAPT-ASl is an 840-bp lncRNA transcribed from the anti-sense strand of the $M A P T$ promoter, and has been identified as a potential epigenetic regulator of $M A P T$ expression in Parkinson's disease (34). However, to the best of our knowledge, there have been no reports of the association between MAPT-AS1 and tumorigenesis to date. Furthermore, the biological functions of LINC00667 and LINC00938 remain to be elucidated. To date, to the best of our knowledge, there is no indication as to why the four-lncRNA signature may serve as a prognostic marker. IncRNAs function in complex ways, and the potential association between these molecules are crucial to understanding their underlying mechanisms of action.

In the present study, differential expression analysis was performed on gene expression data from TCGA and GEO databases. Compared with non-cancerous samples, $P V T 1, M A P T-A S 1$ and LINC00667 were upregulated, while LINC00938 was downregulated in breast cancer tissues. Therefore, PVT1, MAPT-ASI and LINC00667 were considered to be candidate oncogenes, while LINC00938 may serve as a cancer-suppressor gene. Moreover, GO and KEGG analyses were employed to investigate the potential functions of the four lncRNAs. The results showed that PVT1 and $L I N C 00667$ were associated with transcription regulation, while MAPT-AS1, LINC00667 and LINC00938 were associated with cellular mitosis, and PVT1 was associated with mitochondrial energy metabolism. These fundamental biological processes are essentially involved in tumorigenesis and cancer progression $(35,36)$. Additionally, KEGG analysis indicated that LINC00667 was associated with the 'Notch signaling pathway', and previous study has demonstrated that dysregulated Notch signaling is oncogenic, inhibits apoptosis and promotes cell survival (37).

In conclusion, the present study identified a four-lncRNA signature with predictive value for breast cancer prognosis, which may be used as a novel biomarker for the prognosis of patients with breast cancer. Although the signature may contribute to the prognostic evaluation of breast cancer, one of the limitations of the present study is that it was a bioinformatics analysis, and therefore further studies are required using clinical samples, in order to evaluate the identified four-lncRNA signature, in addition to determining the functional mechanisms of these lncRNAs.

\section{Acknowledgements}

Not applicable.

\section{Funding}

No funding was received.

\section{Availability of data and materials}

The breast cancer gene expression data, together with the corresponding clinical information are available from TCGA (https://cancergenome.nih.gov/). The dataset GSE5764 was downloaded from the GEO database (https://www.ncbi.nlm. nih.gov/geo/). The data of associated genes and pathways for GO and KEGG analyses are available in the DAVID database (https://david.ncifcrf.gov/). 


\section{Authors' contributions}

JW and MZ designed the study and conducted bioinformatic analysis. QL, HH, DP, CS and MZ sorted the data and participated in the statistical analysis. MZ drafted the manuscript. DP and CS participated in drafting the manuscript and providing research guidance. JW reviewed and edited the manuscript. All authors read and approved the final manuscript. All authors agreed to be accountable for all aspects of the work in ensuring that questions related to the accuracy or integrity of any part of the work are appropriately investigated and resolved.

\section{Ethics approval and consent to participate}

Not applicable.

\section{Patient consent for publication}

Not applicable.

\section{Competing interests}

The authors declare that they have no competing interests.

\section{References}

1. Siegel RL, Miller KD and Jemal A: Cancer statistics, 2017. CA Cancer J Clin 67: 7-30, 2017.

2. Michaelson JS, Silverstein M, Wyatt J, Weber G, Moore R, Halpern E, Kopans DB and Hughes K: Predicting the survival of patients with breast carcinoma using tumor size. Cancer 95: 713-723, 2002.

3. Rakha EA, El-Sayed ME, Menon S, Green AR, Lee AH and Ellis IO: Histologic grading is an independent prognostic factor in invasive lobular carcinoma of the breast. Breast Cancer Res Treat 111: 121-127, 2008.

4. Shokouh TZ, Ezatollah A and Barand P: Interrelationships Between Ki67, HER2/neu, p53, ER, and PR Status and their associations with tumor grade and lymph node involvement in breast carcinoma subtypes: Retrospective-observational analytical study. Medicine (Baltimore) 94: e1359, 2015.

5. Ma J, Luo DX, Huang C, Shen Y, Bu Y, Markwell S, Gao J, Liu J, Zu X, Cao Z, et al: AKR1B10 overexpression in breast cancer: Association with tumor size, lymph node metastasis and patient survival and its potential as a novel serum marker. Int J Cancer 131: E862-E871, 2012.

6. Crabb SJ, Bajdik CD, Leung S, Speers CH, Kennecke H, Huntsman DG and Gelmon KA: Can clinically relevant prognostic subsets of breast cancer patients with four or more involved axillary lymph nodes be identified through immunohistochemical biomarkers? A tissue microarray feasibility study. Breast Cancer Res 10: R6, 2008.

7. M Braden A, V Stankowski R, M Engel $J$ and A Onitilo A: Breast cancer biomarkers: Risk assessment, diagnosis, prognosis, prediction of treatment efficacy and toxicity, and recurrence. Curr Pharm Des 20: 4879-4898, 2014.

8. Nagini S: Breast Cancer: Current molecular therapeutic targets and new players. Anticancer Agents Med Chem 17: 152-163, 2017.

9. ENCODEProjectConsortium; Birney E,Stamatoyannopoulos JA, Dutta A, Guigó R, Gingeras TR, Margulies EH, Weng Z, Snyder M, Dermitzakis ET, et al: Identification and analysis of functional elements in $1 \%$ of the human genome by the ENCODE pilot project. Nature 447: 799-816, 2007.

10. Clark MB, Johnston RL, Inostroza-Ponta M, Fox AH, Fortini E, Moscato P, Dinger ME and Mattick JS: Genome-wide analysis of long noncoding RNA stability. Genome Res 22: 885-898, 2012.

11. Prensner JR and Chinnaiyan AM: The emergence of lncRNAs in cancer biology. Cancer Discov 1: 391-407, 2011.

12. Rinn JL and Chang HY: Genome regulation by long noncoding RNAs. Annu Rev Biochem 81: 145-166, 2012.

13. Nagano T and Fraser P: No-nonsense functions for long noncoding RNAs. Cell 145: 178-181, 2011.
14. Yoon JH, Abdelmohsen K, Srikantan S, Yang X, Martindale JL, De S,Huarte M,Zhan M, Becker KG and Gorospe M: LincRNA-p21 suppresses target mRNA translation. Mol Cell 47: 648-655, 2012.

15. Peng WX, Koirala P and Mo YY: LncRNA-mediated regulation of cell signaling in cancer. Oncogene 36: 5661-5667, 2017.

16. Bhan A, Soleimani M and Mandal SS: Long noncoding RNA and cancer: A new paradigm. Cancer Res 77: 3965-3981, 2017.

17. Sanchez Calle A, Kawamura Y, Yamamoto Y, Takeshita F and Ochiya T: Emerging roles of long non-coding RNA in cancer. Cancer Sci 109: 2093-2100, 2018.

18. Gupta RA, Shah N, Wang KC, Kim J, Horlings HM, Wong DJ, Tsai MC, Hung T, Argani P, Rinn JL, et al: Long non-coding RNA HOTAIR reprograms chromatin state to promote cancer metastasis. Nature 464: 1071-1076, 2010.

19. Gutschner T, Hammerle M and Diederichs S: MALAT1-a paradigm for long noncoding RNA function in cancer. J Mol Med (Berl) 91: 791-801, 2013.

20. Chandra Gupta S and Nandan Tripathi Y: Potential of long non-coding RNAs in cancer patients: From biomarkers to therapeutic targets. Int J Cancer 140: 1955-1967, 2017.

21. Turashvili G, Bouchal J, Baumforth K, Wei W, Dziechciarkova M, Ehrmann J, Klein J, Fridman E, Skarda J, Srovnal J, et al: Novel markers for differentiation of lobular and ductal invasive breast carcinomas by laser microdissection and microarray analysis. BMC Cancer 7: 55, 2007.

22. Hortobagyi GN, Edge SB and Giuliano A: New and Important Changes in the TNM staging system for breast cancer. Am Soc Clin Oncol Educ Book 38: 457-467, 2018.

23. Chen G, Wang Z, Wang D, Qiu C, Liu M, Chen X, Zhang Q, Yan G and Cui Q: LncRNA Disease: A database for long-non-coding RNA-associated diseases. Nucleic Acids Res 41 (Database Issue): D983-D986, 2013.

24. Zou AE, Ku J,Honda TK, Yu V, Kuo SZ, Zheng H, Xuan Y, Saad MA, Hinton A, Brumund KT, et al: Transcriptome sequencing uncovers novel long noncoding and small nucleolar RNAs dysregulated in head and neck squamous cell carcinoma. RNA 21: 1122-1134, 2015.

25. Ylipää A, Kivinummi K, Kohvakka A, Annala M, Latonen L, Scaravilli M, Kartasalo K, Leppänen SP, Karakurt S, Seppälä J, et al: Transcriptome sequencing reveals PCAT5 as a novel ERG-regulated long noncoding RNA in prostate cancer. Cancer Res 75: 4026-4031, 2015.

26. Gutschner T and Diederichs S: The hallmarks of cancer: A long non-coding RNA point of view. RNA Biol 9: 703-719, 2012.

27. Gibb EA, Brown CJ and Lam WL: The functional role of long non-coding RNA in human carcinomas. Mol Cancer 10: 38, 2011.

28. Song CV, Teo SH, Taib NA and Yip CH: Surgery for BRCA, TP53 and PALB2: A literature review. Ecancermedicalscience 12: 863, 2018.

29. Sundara Rajan S, Hanby AM, Horgan K, Thygesen HH and Speirs V: The potential utility of geminin as a predictive biomarker in breast cancer. Breast Cancer Res Treat 143: 91-98, 2014.

30. Sørensen KP, Thomassen M, Tan Q, Bak M, Cold S, Burton M, Larsen MJ and Kruse TA: Long non-coding RNA HOTAIR is an independent prognostic marker of metastasis in estrogen receptor-positive primary breast cancer. Breast Cancer Res Treat 142: 529-536, 2013.

31. Yang YF, Liao YY, Yang M, Peng NF, Xie SR and Xie YF: Discordances in ER, PR and HER2 receptors between primary and recurrent/metastatic lesions and their impact on survival in breast cancer patients. Med Oncol 31: 214, 2014.

32. Wilusz JE, Sunwoo H and Spector DL: Long noncoding RNAs: Functional surprises from the RNA world. Genes Dev 23: 1494-1504, 2009.

33. Tseng YY, Moriarity BS, Gong W, Akiyama R, Tiwari A, KawakamiH,Ronning P, Reuland B, GuentherK,Beadnell TC, etal: PVT1 dependence in cancer with MYC copy-number increase. Nature 512: 82-86, 2014.

34. Coupland KG,Kim WS, Halliday GM,Hallupp M,Dobson-Stone C and Kwok JB: Role of the long non-coding RNA MAPT-AS1 in regulation of microtubule associated protein tau (MAPT) expression in Parkinson's disease. PLoS One 11: e 0157924, 2016.

35. Bradner JE, Hnisz D and Young RA: Transcriptional Addiction in Cancer. Cell 168: 629-643, 2017.

36. Valcarcel-Jimenez L, Gaude E, Torrano V, Frezza C and Carracedo A: Mitochondrial Metabolism: Yin and yang for tumor progression. Trends Endocrinol Metab 28: 748-757, 2017.

37. Aithal MG and Rajeswari N: Role of Notch signalling pathway in cancer and its association with DNA methylation. J Genet 92: 667-675, 2013.

This work is licensed under a Creative Commons Attribution-NonCommercial-NoDerivatives 4.0 International (CC BY-NC-ND 4.0) License. 